\title{
Quasilinear Choquard equations involving N-Laplacian and critical exponential nonlinearity
}

sreenadh konijeti ${ }^{1}$, Sarika Goyal ${ }^{2}$, and Reshmi Biswas ${ }^{1}$

${ }^{1}$ Indian Institute of Technology Delhi

${ }^{2}$ Bennett University

December 11, 2021

\begin{abstract}
In the present paper, we study a class of quasilinear Choquard equations involving N-Laplacian and the nonlinearity with the critical exponential growth. We discuss the existence of positive solutions of such equations.

\section{Hosted file}

SSR-final.pdf available at https://authorea.com/users/37506/articles/548973-quasilinearchoquard-equations-involving-n-laplacian-and-critical-exponential-nonlinearity
\end{abstract}

